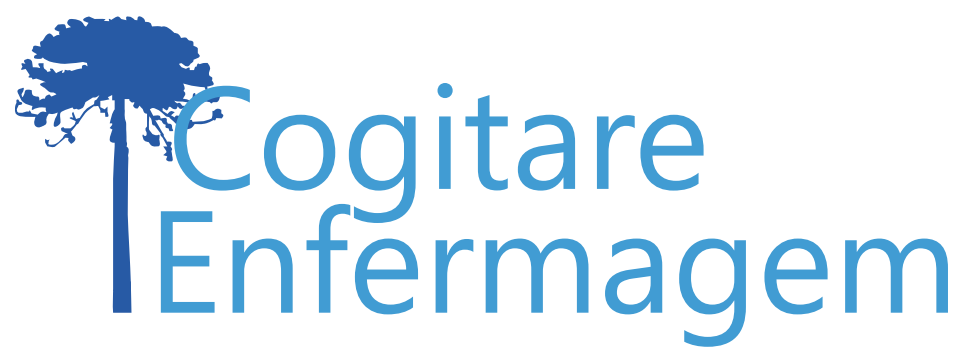

\title{
CUIDADORES DOMICILIARES DE PESSOAS COM DEFICIÊNCIA INTELECTUAL NO CONTEXTO RURAL E SEUS DESAFIOS
}

\author{
HOME-BASED CAREGIVERS OF PEOPLE WITH INTELLECTUAL \\ DISABILITIES IN RURAL SETTINGS AND THEIR CHALLENGES
}

\author{
Tauana Bueno de Quadros da Silva ${ }^{1}$ [i] \\ Darielli Gindri Resta Fontana ${ }^{1}$ (i) \\ Leonardo Bigolin Jantsch ${ }^{1}$ (i) \\ Isabel Cristina dos Santos Colomé ${ }^{1}$ \\ Marta Cocco da Costa ${ }^{1}$ (i)
}

\begin{abstract}
Objective: to analyze the challenges, characteristics, and feelings of home caregivers of people with intellectual disabilities in the rural context. Method: this is a quanti-qualitative study with 124 caregivers of people with intellectual disabilities living in rural settings in eight municipalities in southern Brazil. Data were collected from September/2018 to June/2019, through a questionnaire with social, demographic, and daily variables of care. The analysis was analytical and descriptive type, through frequency and frequency comparison. Results: the home caregivers are women, mothers, sisters, of advanced age, with little education, but who, in the face of challenges such as accumulation of functions, work overload, low education, fatigue, social and professional isolation, develop their attributions with love. Conclusion: the study contributed to the identification of the characteristics and challenges of caregivers of people with intellectual disabilities in rural areas, revealing issues for the advancement of public health practices.
\end{abstract}

DESCRIPTORS: Caregivers; Intellectual Disability; Nursing; Rural Population; Social Support.

COMO REFERENCIAR ESTE ARTIGO:

Silva TB de $Q$ da, Fontana DGR, Jantsch LB, Colomé IC dos S, Costa MC da. Cuidadores domiciliares de pessoas com deficiência intelectual no contexto rural e seus desafios. Cogit. Enferm. [Internet]. 2021 [acesso em "colocar data de acesso, dia, mês abreviado e ano"]; 26. Disponível em: http://dx.doi.org/10.5380/ce.v26i0.72567. 
O cuidador é compreendido como aquele responsável por cuidar de alguém que esteja doente e/ou dependente, auxiliando no desempenho das atividades de vida diária, como alimentação, higiene, lazer, medicações e acompanhamento nos serviços de saúde ${ }^{(1)}$. Além disso, o cuidador é considerado a ponte ou o elo entre quem é cuidado e os serviços, especialmente relativos às questões de saúde ${ }^{(2-3)}$.

O cuidador domiciliar ou informal é aquele que aprendeu com a prática, sem formação para desempenhar tais tarefas, comumente um membro da família. Nesse sentido, percebese que os cuidadores domiciliares passam por situações difíceis, em virtude da ausência de conhecimentos científicos, desenvolvendo um cuidado mais instintivo e baseado na experiência. Cuidar de pessoas com deficiência no domicílio é uma atribuição desafiadora, que envolve amor, respeito, zelo, dedicação e presença, e pode causar desgastes dependendo das condições e características de cada pessoa com deficiência e família ${ }^{(1,4)}$.

A deficiência intelectual é definida pela American Association on Developmental Desabilities (AAIDD) como a coexistência de limitações consideráveis no funcionamento intelectual e déficits de ações adaptativas em pelo menos três áreas, expressa em capacidades conceituais, sociais e práticas, que envolvem atividades diárias do sujeito e sua adaptação às demandas da sociedade ${ }^{(5-6)}$. A deficiência intelectual, por vezes, estabelece uma condição limitada de expressão de vontade ou tomada de decisão do sujeito, por mais que sejam pessoas adultas. Tal limitação torna essas pessoas mais dependentes, seja do cuidador domiciliar, da família ou da equipe de saúde. A deficiência intelectual atinge cerca de 2,6 milhões de pessoas no Brasil; destes, mais de 445 mil residem no meio rural|(7-8).

O cuidador domiciliar de Pessoas com Deficiência Intelectual (PCDI) geralmente vivencia acúmulos de funções inerentes ao processo de estar no domicílio, comumente não tem com quem dividir as atividades de cuidados e acaba realizando múltiplas funções no lar, como a responsabilidade pelas refeições, organização da casa, cuidados com filhos, atividade profissional. Esse contexto gera sobrecarga, esgotamento, isolamento afetivo e social. Além disso, o fato de ser rotineiro pode ocasionar ou agravar doenças no cuidador $^{(9-10)}$. Somados a isso, aparecem outros fatores que contribuem para a sobrecarga como a idade, as condições socioeconômicas e do ambiente, assim como os determinantes sociais (saneamento básico, lazer, cultura, alimentação e outros) ${ }^{(11)}$. As características do rural acabam privando as populações desses recursos, podendo dificultar o processo de universalização de direitos e implicando na qualidade de vida das famílias ${ }^{(12-13)}$.

Os indicadores de saúde e os determinantes sociais da população rural, em geral, são menores que os da população urbana, além da cobertura de ações de prevenção, também menos frequente na área rural. As dificuldades relacionadas aos serviços de saúde ocorrem por conta de obstáculos como inserção e manutenção dos profissionais nessas áreas, rotatividade dos trabalhadores e distribuição desigual por vezes, o que também acarreta em sobrecarga de trabalho para os profissionais ${ }^{(13-14)}$.

A deficiência intelectual por si só já causa grandes dificuldades e limitações para quem reside no rural, pois as pessoas que vivem no campo e não tem deficiência referem ter menos dificuldades quando comparadas com as que residem no campo e tem deficiência, mostrando que a deficiência é um fator agravante para limitações e dificuldades enfrentadas por essa população diariamente ${ }^{(15)}$.

Existem poucos estudos voltados para o cuidador de PCDI no cenário rural. Considerase um passo importante nesse cenário romper com a invisibilidade, reconhecer suas especificidades e se aproximar desse contexto(11,15-16). Portanto, identificar e compreender os desafios vividos pelos cuidadores domiciliares da PCDI pode contribuir com melhorias no processo de cuidar no domicílio, especialmente no cenário rural. O objetivo deste estudo foi analisar os desafios, características e sentimentos de cuidadores domiciliares de 
pessoas com deficiência intelectual no contexto rural.

\section{MÉTODO}

Este estudo caracteriza-se como um recorte do banco de dados do projeto matricial "Determinantes sociais de saúde em pessoas com deficiência, famílias e rede de apoio no cenário rural: múltiplas vulnerabilidades". O projeto matricial entrevistou 275 pessoas com deficiência e seus cuidadores, em oito municípios, com característica rural, na região noroeste do estado do Rio Grande do Sul, Brasil. O presente manuscrito fez recorte dos cuidadores de PCDI, totalizando 124 participantes.

Trata-se de um estudo quanti-qualitativo realizado com cuidadores domiciliares de PCDI, O critério utilizado para a seleção dos municípios do estudo foi ter, no mínimo, 70\% da população residindo no meio rural, totalizando oito municípios.

Os critérios de seleção foram cuidadores domiciliares de PCDI maiores de 18 anos e que não possuíam deficiência mental ou intelectual. Os participantes foram selecionados e contatados por meio dos registros disponíveis nos Serviços Municipais de Saúde, através do contato prévio dos pesquisadores com os enfermeiros das unidades de saúde dos municípios.

O trabalho de campo consistiu em, primeiramente, selecionar as PCDI que viviam no rural e, posteriormente, a definição dos principais cuidadores dessa população. $O$ agente comunitário de saúde (ACS) foi o profissional responsável pela interação dos pesquisadores com a população do estudo (cuidadores). Após o contato inicial com os serviços de saúde dos municípios participantes, as entrevistas foram agendadas, de acordo com a disponibilidade dos ACS e dos cuidadores, conforme datas de coletas disponibilizadas pelos pesquisadores.

As informações foram coletadas através de um questionário próprio, com as seguintes questões abertas: a função de cuidador desperta quais sentimentos em você? Quais são as principais dificuldades que você sente no cuidado à pessoa com deficiência? Também foram construídas, por meio de instrumento fechado, a caracterização quanto a idade, sexo, cor e demais variáveis socioeconômicas. O instrumento foi aplicado pelos pesquisadores, no domicílio das pessoas com deficiência intelectual. A coleta aconteceu no período de setembro de 2018 a junho de 2019. Participaram do estudo 124 cuidadores domiciliares de PCDI.

Os dados quantitativos foram digitados em planilhas Excel, posteriormente analisados sob análise descritiva e analítica (comparação de frequência, teste Qui-quadrado e Exato de Fisher), por meio do programa estatístico Statistical Product and Service Solutions (SPSS), versão 18.0. Utilizou nível de significância com valor de p menor ou igual a 5\%. Para análise dos dados qualitativos, utilizou-se o aplicativo Word Cloud ("Nuvem de Palavras"), que organiza/apresenta as palavras com maior repetição (análise de frequência simples) para construção dos resultados, por meio de figuras de destaques. As palavras foram digitadas de acordo com a frequência absoluta de respostas nas entrevistas, e as mais repetidas ganham destaque no tamanho (maior fonte de apresentação) na representação da "nuvem" de palavras. Trata-se de uma análise qualitativa por repetição das palavras chaves elencadas nos enunciados.

O estudo foi aprovado pelo Comitê de Ética em Pesquisa da Universidade Federal de Santa Maria, sob parecer número 2.208.566. 
Os resultados são apresentados em tabelas e nuvens de palavras, buscando caracterizar os cuidadores de PCDI e seus desafios cotidianos. Na Tabela 1, é descrita a caracterização desses cuidadores.

Tabela 1 - Caraterização dos Cuidadores de Pessoas com Deficiência Intelectual. Palmeira das Missões, RS, Brasil, 2019

\begin{tabular}{|c|c|c|}
\hline Variável & $\mathbf{n}$ & $\%$ \\
\hline \multicolumn{3}{|l|}{ Grau de Parentesco } \\
\hline Pai & 8 & 6,5 \\
\hline Mãe & 55 & 44,4 \\
\hline Irmão & 29 & 23,4 \\
\hline Tio ou Tia & 3 & 2,4 \\
\hline Outros & 29 & 23,4 \\
\hline \multicolumn{3}{|l|}{ Sexo } \\
\hline Masculino & 25 & 20,2 \\
\hline Feminino & 99 & 79,8 \\
\hline \multicolumn{3}{|l|}{ Idade } \\
\hline 18-42 anos & 30 & 24,2 \\
\hline 43-52 anos & 33 & 26,6 \\
\hline $53-63$ anos & 30 & 24,2 \\
\hline$>63$ anos & 31 & 25 \\
\hline \multicolumn{3}{|l|}{ Cor } \\
\hline Branca & 90 & 72,6 \\
\hline Parda & 31 & 25 \\
\hline Negra & 2 & 1,6 \\
\hline Outra & 1 & 0,8 \\
\hline \multicolumn{3}{|l|}{ Escolaridade } \\
\hline Não Frequentou Escola & 9 & 7,3 \\
\hline Ens. Fund. Incompleto & 86 & 69,4 \\
\hline Ens. Fund. Completo & 7 & 5,6 \\
\hline Ens. Méd. Incompleto & 7 & 5,6 \\
\hline Ens. Méd. Completo & 10 & 8,1 \\
\hline Superior Incompleto & 3 & 2,4 \\
\hline Superior Completo & 2 & 1,6 \\
\hline \multicolumn{3}{|l|}{ Renda } \\
\hline Até um salário-mínimo & 42 & 33,9 \\
\hline Dois a cinco salários & 82 & 66,1 \\
\hline
\end{tabular}

Fonte: Autores (2019) 
Conforme a Tabela 1, 94 (75\%) cuidadores possuíam mais de 43 anos de idade, a média de idade foi de 52,5 anos, mínimo de 18 anos e máximo de 82 anos. Quanto ao grau de parentesco, $55(44,4 \%)$ cuidadores de PCDI eram as mães, seguidos dos irmãos ( $n=29$, $23,4 \%)$. Os cuidadores são do sexo feminino $(n=99 ; 79,8 \%)$, de cor branca $(n=90 ; 72,6 \%)$ e com renda familiar de dois a cinco salários-mínimos ( $n=82 ; 66,1 \%)$. Sob uma análise de graus de instrução, destaca-se a baixa escolaridade, visto que 95 (76,7\%) dos cuidadores nunca estudaram ou tinham ensino fundamental incompleto.

Sob uma análise do desenvolvimento de doenças nos cuidadores, destaca-se que 106 $(85,5 \%)$ cuidadores de PCDI convivem com alguma doença crônica. A Tabela 2 compara alguns fatores associados ao desenvolvimento desses agravos, antes e após tornar-se cuidador de uma PCDI.

Tabela 2 - Fatores associados ao desenvolvimento de doenças nos cuidadores de pessoas com deficiência intelectual que residem no meio rural na região noroeste do estado do Rio Grande do Sul $(n=106)$. Palmeira das Missões, RS, Brasil, 2019

\section{Doença Prévia Doença Adquirida p-valor}

\begin{tabular}{|c|c|c|c|}
\hline \multicolumn{4}{|l|}{ Tipo de Deficiência } \\
\hline Deficiência intelectual & $22(34,4)$ & $11(26,2)$ & \multirow[t]{2}{*}{$0,04^{*}$} \\
\hline Outros tipos de deficiência & $42(65,6)$ & $31(73,8)$ & \\
\hline \multicolumn{4}{|l|}{ Sexo } \\
\hline Masculino & $12(18,7)$ & $9(21,4)$ & \multirow[t]{2}{*}{$0,496^{*}$} \\
\hline Feminino & $52(81,3)$ & $33(78,6)$ & \\
\hline \multicolumn{4}{|l|}{ Membro familiar } \\
\hline Pai & $5(7,8)$ & $4(9,5)$ & \multirow[t]{5}{*}{$0,004^{\star}$} \\
\hline Mãe & $31(48,4)$ & $26(61,9)$ & \\
\hline Irmão & $13(20,3)$ & $7(16,6)$ & \\
\hline Tio/Tia & $2(3,1)$ & 0 & \\
\hline Outros & $13(20,3)$ & $5(11,9)$ & \\
\hline \multicolumn{4}{|l|}{ Descanso } \\
\hline Nunca descansa & $9(14,1)$ & $4(9,5)$ & \multirow[t]{4}{*}{$0,004^{*}$} \\
\hline Menos de um turno & $3(4,7)$ & $3(7,1)$ & \\
\hline Um turno & $14(21,9)$ & $9(21,4)$ & \\
\hline Somente à noite & $38(59,4)$ & $26(61,9)$ & \\
\hline TOTAL & $64(100)$ & $42(100)$ & \\
\hline
\end{tabular}

*Teste Qui-quadrado

Fonte: Autores (2019)

Conforme a Tabela 2, cuidadores de pessoas com deficiência intelectual foram menos doentes previamente e apresentaram menores taxas para o desenvolvimento de doenças $(p=0,04)$, após o diagnóstico de deficiência intelectual, quando comparados a cuidadores de pessoas com outros tipos de deficiência. Daqueles cuidadores que desenvolveram doença após o diagnóstico, foi mais frequente quando eram mães, quando comparados a 
demais membros da família $(p=0,004)$. Vale destacar que não houve diferença significativa entre sexo, do cuidador $(p=0,496)$ para desenvolvimento de doenças após iniciar os cuidados. É importante ressaltar que os cuidadores descansam apenas à noite e que, aproximadamente, um em cada 10 nunca descansa.

Em uma análise sobre suporte social e rede de apoio/informações dos cuidadores, destaca-se que $72(54,1 \%)$ disseram que receberam algum tipo de informação para o cuidado das PCD. Frequentemente, os responsáveis pelas informações foram os Serviços de Saúde $(52,9 \%, n=27)$ e a Associação de Pais e Amigos dos Excepcionais (APAE) $(25,5 \%$, $\mathrm{n}=13)$. As atividades sociais/recreativas de metade dos cuidadores $(50 \% \mathrm{n}=62)$ se dá pela participação em grupos na comunidade, sendo no mínimo um grupo.

Os sentimentos e os desafios dos cuidadores de PCDI foram analisados de forma qualitativa, construindo duas nuvens de palavras. Com relação aos sentimentos despertados no cuidador em relação ao cuidado desenvolvido, conforme a Figura 1, a palavra mais destacada foi felicidade, seguida por responsabilidade e amor. Também foram mencionadas palavras como raiva, cansaço, preocupação, pena e tristeza.

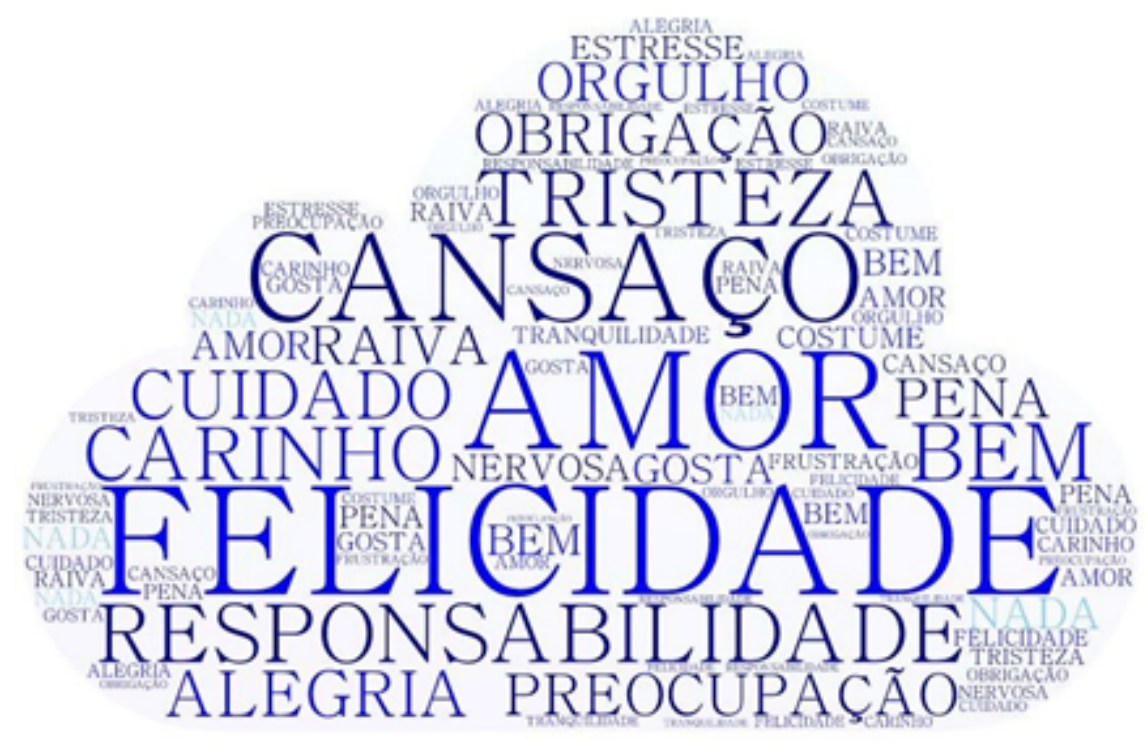

Figura 1 - Representação e sentimentos de cuidadores de PCDI no cotidiano de cuidado. Palmeira das Missões, RS, Brasil, 2019

Fonte: Autores (2019)

Com relação às dificuldades e desafios no cotidiano de cuidado, as palavras que ganharam destaque foram: dependência, agressividade, cuidados da vida diária (CVD), renda, falta de informação, acesso a saúde, preocupação, medo; e alguns mencionaram que não vivenciam dificuldades. A Figura 2 representa essa descrição. 


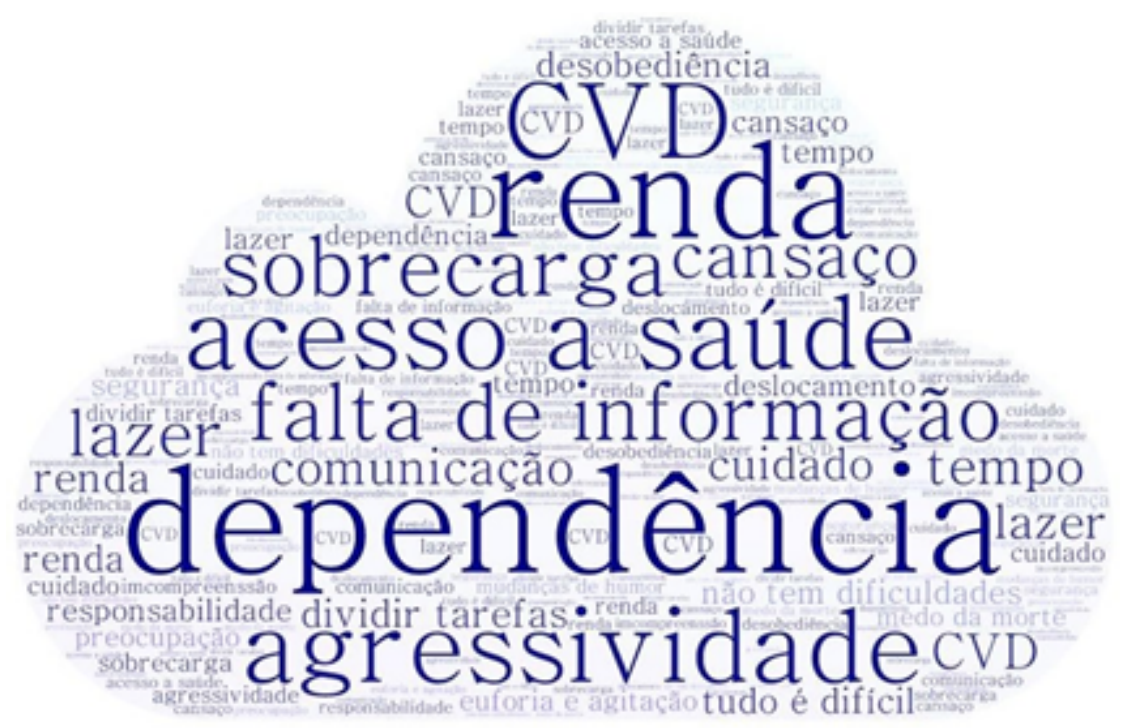

Figura 2 - Dificuldades e Desafios de cuidadores de PCDI. Palmeira das Missões, RS, Brasil, 2019 Fonte: Autores (2019)

As cuidadoras são mulheres (cerca de $80 \%$ ) - esse achado concorda que histórica e socialmente, a mulher é tida como cuidadora, seja do domicílio ou da saúde de membros da família, o que pode ser observado em outras pesquisas ${ }^{(17-19)}$. Quanto ao grau de parentesco, geralmente são mães e irmãs, pois, frequentemente, elas tomam para si a atribuição de cuidar dos seus familiares ${ }^{(20)}$. A esse cuidado somam-se tarefas do domicílio, cuidado com os filhos e cuidado de si, acumulando responsabilidades e sobrecarga física relacionada à dupla jornada de trabalho. Muitas mulheres, nas diferentes realidades já estudadas, apresentam cansaço físico, emocional e mental, desenvolvimento de transtornos mentais como estresse, ansiedade e depressão, motivos para o uso de medicações, pois não podem optar pelo que melhor se encaixa na sua realidade ${ }^{(21-22)}$.

Em relação à escolaridade, $95(76,7 \%)$ cuidadores não frequentaram a escola ou não concluíram o ensino fundamental, assim como as PCDI: $91 \%$ não frequentaram a escola ou não concluíram o ensino fundamental. O nível de escolaridade se constitui como indicador de vulnerabilidade social, tanto do cuidador como de quem é cuidado. A baixa escolaridade colabora na redução do avanço social e econômico, podendo condicionar fragilidades sociais e precarização nos acessos às necessidades da vida diária. É ainda uma situação que interfere na compreensão dos direitos e das possibilidades de cuidado, garantidos por políticas públicas. A vulnerabilidade social é um dos elementos que favorecem a insuficiência de recursos individuais, familiares e sociais para assistir às demandas das famílias ${ }^{(19,23)}$.

Percebe-se que são pessoas com grau de escolaridade baixo, cuidando de pessoas na mesma condição. A baixa escolaridade afeta o cuidado, pode causar dificuldade no entendimento da informação e na capacidade de desenvolver as tarefas, afetar a renda e a produtividade, além de comprometer as responsabilidades do cuidador para com quem é cuidado, uma vez que a realização do cuidado compreende a administração de medicamentos, as orientações técnico-científicas e a busca por melhor qualidade de vida para ambos ${ }^{(19-23)}$.

Como a expectativa de vida vem aumentando, o número de idosos cuidando de outros idosos tende a aumentar também, visto que a média de idade dos cuidadores foi de 52 anos. Geralmente, a tarefa de cuidar decorre do nível de saúde do cuidador e de 
quem cuida, apoio recebido, prognóstico da doença de quem é cuidado e a qualidade nas convivências familiares (relações entre quem cuida e é cuidado). Todas essas características remetem ao cuidador mais experiente, e de melhor relação com membros familiares ${ }^{(23)}$. $O$ acúmulo de atribuições é uma das dificuldades encontradas pelo cuidador, principalmente na velhice, porém, muitos cuidadores aprendem a lidar bem e a conciliar suas atividades da vida diária com as demandas de cuidar do outro sem se sentirem prejudicados ${ }^{(24)}$.

Quando a limitação cognitiva da PCDI influencia na sua independência, a perda de funções motoras ou o surgimento de alguma doença crônica no cuidador dificulta as ações de cuidados $^{(25)}$. O cuidador idoso é suscetível à instalação de doenças próprias da velhice e o cuidar, muitas vezes, o coloca como um doente em iminência, com capacidade operacional em risco ${ }^{(24)}$.

Os participantes deste estudo, quando comparados com cuidadores de pessoas com outros tipos de deficiências, apresentaram menores taxas de desenvolvimento de doenças após tornarem-se cuidadores, o que pode estar associado a condições de esforço físico e/ou limitação funcional (pouco presentes nos PCDI) com as doenças ergonômicas e posturais $^{(18)}$.

A sobrecarga foi uma das dificuldades relatadas pelos entrevistados, sendo um obstáculo significativo no cotidiano do cuidador domiciliar. Ela pode ser definida pela redução do sentimento de bem-estar e ocorrência de complicações de saúde, que podem aparecer de forma aguda ou crônica. Exercer a função de cuidador domiciliar afeta a saúde física e emocional, principalmente quando o mesmo possuía algum problema de saúde antes de se tornar cuidador. Observa-se a importância de compartilhar as atividades acerca dos cuidados com a PCDI e receber orientações para otimizar o tempo e evitar desgastes ${ }^{(26)}$.

Os desafios vivenciados por cuidadores domiciliares no espaço rural são inúmeros: principalmente barreiras relacionadas à acessibilidade, serviços de saúde, saneamento básico, lazer, distanciamento geográfico, isolamento profissional e familiar, entre outras. Além disso, a cobertura dos serviços de saúde para populações rurais é uma dificuldade mundial, demostrando os piores indicadores de saúde nestas áreas ${ }^{(13)}$. Uma estratégia que pode contribuir é investir no desenvolvimento de relações sociais intrafamiliares e extrafamiliares. É importante existir uma estrutura familiar que possibilite o envolvimento entre o cuidador domiciliar e a PCDI dentro do espaço familiar, promovendo um ambiente seguro, calmo e receptivo. Da mesma forma, é necessário que haja socialização, interações e habilidades sociais em outros contextos, visto que são indicadores de saúde mental, relações interpessoais saudáveis, bases da vida em sociedade, além de reduzir as fragilidades do cuidar e estimular a interação e troca de saberes ${ }^{(27)}$.

A sobrecarga tem relação com o nível de dependência da PCDI, e pode estar relacionada com a falta de compreensão acerca dos desafios e das necessidades cotidianas da família, caracterizadas, muitas vezes pela presença de violência, agressividade, medo, raiva e sensação de estar sozinho nesse processo, especialmente na ruralidade. Para amenizar essas fragilidades e enfrentar os desafios, os profissionais de saúde e os cuidadores precisam se encontrar, construir relações dialógicas e propositivas, proporcionando qualidade de vida, diminuindo sobrecargas e aprimorando os cuidados realizados. A sobrecarga afeta também as relações sociais dos cuidadores, uma vez que a falta de apoio emocional, falta de tempo devido à rotina de cuidados e às necessidades da PCDI podem influenciar nas relações afetivas, e gerar isolamento social para o cuidador $^{(28)}$.

As necessidades de quem reside no rural são peculiares e requerem cuidado de diferentes serviços, principalmente do campo da saúde ${ }^{(12)}$. Os serviços de saúde têm papel fundamental para o desenvolvimento das ações de cuidado prestadas pelo cuidador no domicílio, especialmente acolhendo de maneira sensível as necessidades e dificuldades do cuidador de PCDI. Os frutos da interação cuidador-profissional são percebidos por intermédio de uma relação dialógica, da efetividade nas soluções de problemas, do envolvimento e do compartilhamento de objetivos, da construção de estratégias de 
cuidados singulares e da promoção de positivos e contínuos encontros ${ }^{(29)}$.

$O$ apoio que o cuidador domiciliar e a PCDI recebem de familiares, amigos e profissionais reflete significativamente no bem-estar, na confiança e no clima de relacionamento entre os dois, uma vez que a carga física, psicológica e emocional do cuidador é alta. Mesmo que predominem aspectos positivos entre os relacionamentos de quem cuida e é cuidado, os desgastes físicos e emocionais existem ${ }^{(30)}$.

As redes de apoio podem ser tanto formais (políticas públicas, previdência e assistência social, entre outras), quanto informais (família, vizinhos, amigos e comunidade) (24). As redes de apoio contribuem na elaboração dos sentimentos que a função de cuidador desperta e encoraja o enfrentamento dos desafios.

Reconhece-se como limitação do estudo a descrição locorregional de uma população específica, com a seleção populacional baseada em relato dos serviços de saúde, o que pode ter excluído PCDI desconhecidas pelos trabalhadores de saúde dos municípios estudados.

\section{CONCLUSÃO}

Mulheres, mães, adultas e idosas, com fragilidades de muitos acessos (entre eles, escola), renda, apoio social, profissional e, principalmente, vivendo contextos de agressividade, tensão e solidão são algumas das características dessas cuidadoras que vivem no rural.

O fortalecimento de Políticas Públicas para quem reside no campo, a implantação da Estratégia de Saúde da Família Rural, a realização de visitas domiciliares e grupos de saúde na comunidade são meios importantes de qualificar os cuidadores, esclarecer questões de saúde, promover vínculo, qualificar o cuidado, diminuir sobrecargas e compreender as vivências desses cuidadores. Observou-se a relevância da equipe de saúde nesse contexto, fortalecendo as relações que existem entre o cuidador/PCDI/família, garantindo autonomia e qualidade de vida para todos.

Acredita-se que mais estudos devem ser realizados sobre o cuidador que reside no rural e suas demandas, para promover saúde e minimizar agravos e vulnerabilidades. Produções teóricas e práticas precisam ser construídas para diminuir distâncias e contribuir com a ciência e o saber popular acerca do cuidador de PCDI que reside no rural.

O estudo identificou e caracterizou os cuidadores de PCDI no rural e seus desafios, mostrando as fragilidades e as lacunas a serem supridas nos âmbitos teóricos e práticos do campo da saúde.

\section{AGRADECIMENTOS}

À FAPERGS pelo financiamento do Projeto de Pesquisa pela Chamada FAPERGS/ MS/CNPQ/SESRS n. 03/2017 - Programa pesquisa para o SUS: gestão compartilhada em saúde PPSUS - 2017. 
1. Diniz MAA, Melo BR de S, Neri KH, Casemiro FG, Figueiredo LC, Gaiolo CCL de O, et al. Comparative study between formal and informal caregivers o folder adults. Ciênc. Saúde Colet. [Internet]. 2018 [acesso em 10 dez 2019]. 23(11). Disponível em: https://doi.org/10.1590/1413-812320182311.16932016.

2. Matos ACGT, Carvalho ES de S, Passos $S$ da SS, Silva RS da. Family caregivers challenges about caring for children with impaired skin integrity. Esc Anna Nery [Internet]. 2018 [acesso em 12 fev 2020]; 22(4). Disponível em: https://doi.org/10.1590/2177-9465-ean-2018-0173.

3. Almeida MHS, Mendonça E de S. Um olhar à família: ressonâncias psicossociais em familiares que convivem com uma pessoa em situação de transtorno mental. Barbarói [Internet]. 2017 [acesso em 15 abr 2020]; 49. Disponível em: https://online.unisc.br/seer/index.php/barbaroi/article/viewFile/6617/6903.

4. Yavo I de S, Campos EMP. Cuidador e cuidado: o sujeito e suas relações no contexto da assistência domiciliar. Psicol. Teor. Prat [Internet]. 2016 [acesso em 12 dez 2019]; 18(1). Disponível em: http://pepsic. bvsalud.org/scielo.php?script=sci arttext\&pid=S1516-36872016000100002\&lng=pt\&nrm=iso.

5. American Association on Intellectual and Developmental Desabilities (AAIDD). Definition of Intellectual Disability. [Internet]. 2019 [acesso em 15 dez 2019]. Disponível em: https://www.aaidd.org/intellectualdisability/definition.

6. Angonese LS, Boueri IZ, Schmidt A. O adulto com deficiência intelectual: concepção de deficiência e trajetória de carreira. Rev. Bras. Orientac. Prof. [Internet]. 2015 [acesso em 08 jan 2020]; 16(1). Disponível em: http://pepsic.bvsalud.org/scielo.php?script=sci arttext\&pid=S1679-33902015000100004.

7. Instituto Brasileiro de Geografia e Estatística (IBGE). Instituto Brasileiro de Pesquisa e Estatística. Cartilha do Censo 2010. [Internet]. 2012. [acesso em 15 abr 2020]. Disponível em: https://censo2010. ibge.gov.br/.

8. Machado ILO, Albuquerque A. Papel do Estado quanto à vulnerabilidade e proteção de adultos com deficiência intelectual. Cad. Ibero-amer. [Internet]. 2019 [acesso em 10 jan 2020]; 8(1). Disponível em: http://docs.bvsalud.org/biblioref/2019/05/996361/05 papel-do-estado.pdf.

9. Areosa SVC, Henz LF, Lawisch D, Areosa RC. Cuidar de si e do outro: estudo sobre os cuidadores de idosos. Psic. Saúde \& Doenças [Internet]. 2014 [acesso em 12 jan 2020]; 15(2). Disponível em: http://www. scielo.mec.pt/scielo.php?script=sci arttext\&pid=S1645-00862014000200012.

10. Costa TF da, Gomes TM, Viana LR de C, Martins KP, Costa KN de FM. Stroke: patient characteristics and quality of life of caregivers. Rev Bras Enferm [Internet]. 2016. [acesso em 12 jan 2020]; 69(5).

Disponível em: https://doi.org/10.1590/0034-7167-2015-0064.

11. Coimbra Junior CEA. Rural Health in Brazil: a still relevant old subject. Rev. Saúde Pública

[Internet]. 2018. [acesso em 08 jan 2020]. 52(sup/1). Disponível em: https://doi.org/10.11606/s1518-

8787.2018052000supl1ap.

12. Winckler M, Boufleuer TR, Ferreti F, Sá CA de. Idosos no meio rural: uma revisão integrativa. Estud. interdiscipl. envelhec. [Internet]. 2016 [acesso em 11 jan 2020]. 21(2). Disponível em: https://seer.ufrgs.br/ RevEnvelhecer/article/view/60691/44545.

13. Oliveira AR de, Sousa YG de, Diniz IVA, Medeiros SM de, Martiniano C, Alves M. The daily routine of nurses in rural areas in the Family Health Strategy. Rev Bras Enferm [Internet]. 2019 [acesso em 06 jan 2020]; 72(4). Disponível em: https://doi.org/10.1590/0034-7167-2018-0243.

14. Shimizu HE, Trindade J de S, Mesquita MS de, Ramos MC. Evaluation of the Responsiveness Index of the Family Health Strategy in rural areas. Rev Esc Enferm USP. [Internet]. 2018 [acesso em 10 jan 2020]; 52. Disponível em: https://doi.org/10.1590/s1980-220x2017020203316.

15. Ursine BL, Pereira EL, Carneiro FF. Saúde da pessoa com deficiência que vive no campo: o que dizem os trabalhadores da Atenção Básica? Interface (Botucatu). [Internet]. 2018 [acesso em 04 jan 2020]; 22(64). Disponível em: https://doi.org/10.1590/1807-57622016.0666.

16. Lima ARA, Buss E, Ruiz M del CS, González JS, Heck RM. Possibilidades de formação em enfermagem 
rural: revisão integrativa. Acta Paul Enferm. [Internet]. 2019 [acesso em 19 dez 2019]; 32(1). Disponível em: https://doi.org/10.1590/1982-0194201900016.

17. Santos CF, Eulálio M do C, Barros PM. O sentido do cuidar para familiares de pessoas com transtorno mental: um estudo descritivo. Mudanças - Psicologia da Saúde [Internet]. 2015 [acesso em 08 jan 2020]; 23(2). Disponível em: http://dx.doi.org/10.15603/2176-1019/mud.v23n2p27-35.

18. Egan C, Dalton CT. An exploration of care-burden experienced by older caregivers of adults with intellectual disabilities in Ireland. Br J Learn Disabil. [Internet]. 2019 [acesso em 22 nov 2019]; 47(3). Disponível em: https://doi.org/10.1111/bld.12273.

19. Jesus ITM de, Orlandi AA dos S, Zazzetta MS. Burden, profile and care: caregivers of socially vulnerable elderly persons. Rev. Bras. Geriatr. Gerontol [Internet]. 2018 [acesso em 19 nov 2019]; 21(2). Disponível em: https://doi.org/10.1590/1981-22562018021.170155.

20. Souza ID, Pereira J de A, Silva EM. Between State, society and Family: the care off emale caregivers. Rev. Bras. Enferm [Internet]. 2018 [acesso em 14 dez 2019]; 71(suppl6). Disponível em: https://doi. org/10.1590/0034-7167-2018-0111.

21. Meira EC, Reis LA dos, Gonçalves LHT, Rodrigues VP, Philipp RR. Women's experiences in terms of the care provided to dependent elderly: gender orientation for care. Esc Anna Nery [Internet]. 2017 [acesso em 15 abr 2020]; 21(2). Disponível em: https://doi.org/10.5935/1414-8145.20170046.

22. Cezar-Vaz MR, Bonow CA, Abreu DPG, Vaz JC, Mello MCVA de, Xavier DM. Rural workload and factors associated with the use of medication by elderly people. Rev Esc Enferm USP [Internet]. 2018 [acesso em 04 dez 2019]; 52. Disponível em: http://dx.doi.org/10.1590/S1980-220X2017048303374.

23. Almeida L de PB, Menezes TM de O, Freitas AV da S, Pedreira LC. Características sociais e demográficas de idosos cuidadores e motivos para cuidar da pessoa idosa em domicilio. Rev Min Enferm. [Internet]. 2018 [acesso em 6 dez 2019]; 22(1). Disponível em: http://www.dx.doi.org/10.5935/14152762.20180004.

24. Mocelin C, Silva TG da, Celich KLS, Madureira VF, Souza SS de, Colliselli L. The care of dependente elderly on the Family contexto. Rev Fun Care Online [Internet]. 2017. [acesso em 9 dez 2019]; 9(4). Disponível em: http://dx.doi.org/10.9789/2175-5361.2017.v9i4.1034-1039.

25. Santos-Orlandi AA dos, Brito TRP de, Ottaviani AC, Rossetti ES, Zazzetta MS, Gratão ACM, et al. Perfil de idosos que cuidam de outros idosos em contexto de alta vulnerabilidade social. Esc Anna Nery [Internet]. 2017 [acesso em 15 abr 2020]; 21(1). Disponível em: http://www.scielo.br/pdf/ean/v21n1/14148145-ean-21-01-e20170013.pdf.

26. Guerra HS, Almeira NAM, Souza MR de, Minamisava R. A sobrecarga do cuidador domiciliar. Rev Bras Promoç Saúde [Internet]. 2017 [acesso em 26 nov 2019]; 30(2). Disponível em: http://dx.doi. org/10.5020/18061230.2017.p179.

27. Rückert B, Cunha DM, Modena CM. Saberes e práticas de cuidado em saúde da população do campo: revisão integrativa da literatura. Interface (Botucatu). [Internet]. 2018 [acesso em 19 nov 2019]; 22(66). Disponível em: https://doi.org/10.1590/1807-57622017.0449.

28. Couto AM do, Caldas CP, Castro EAB de. Cuidado domiciliar a idosos dependentes de cuidadores familiares com sobrecarga e desconforto emocional. Rev Fun Care Online [Internet]. 2019 [acesso $15 \mathrm{abr}$ 2020]; 11(4). Disponível em: http://www.seer.unirio.br/index.php/cuidadofundamental/article/view/6782/ pdf.

29. Silva RS, Fedosse E. Perfil sociodemográfico e qualidade de vida de cuidadores de pessoas com deficiência intelectual. Cad. Bras. Ter. Ocup. [Internet]. 2018 [acesso em 10 mar 2020]; 26(2). Disponível em: https://doi.org/10.4322/2526-8910.ctoA01220.

30. Mitter N, Ali A, Scior K. Stigma experienced by families of individuals with intellectual disabilities and autism: a systematic review. Research in Developmental Disabilities. [Internet]. 2019 [acesso em 25 out 2019]; 89. Disponível em: https://doi.org/10.1016/j.ridd.2019.03.001. 


\section{CUIDADORES DOMICILIARES DE PESSOAS COM DEFICIÊNCIA INTELECTUAL NO CONTEXTO RURAL E SEUS DESAFIOS}

RESUMO:

Objetivo: analisar os desafios, características e sentimentos de cuidadores domiciliares de pessoas com deficiência intelectual no contexto rural. Método: trata-se de um estudo quanti-qualitativo, com 124 cuidadores de pessoas com deficiência intelectual que vivem no contexto rural, em oito municípios do sul do Brasil. Os dados foram coletados de setembro/2018 a junho/2019, por meio de um questionário com variáveis sociais, demográficas e cotidianas do cuidado. A análise foi do tipo analítica e descritiva, por meio de frequência e comparação de frequência. Resultados: os cuidadores domiciliares são mulheres, mães, irmãs, com idade avançada, pouca escolaridade, mas que, diante dos desafios como acúmulo de funções, sobrecarga de trabalho, baixa escolaridade, cansaço, isolamento social e profissional, desenvolvem suas atribuições com amor. Conclusão: o estudo contribuiu na identificação das características e desafios dos cuidadores de pessoas com deficiência intelectual no espaço rural, revelando questões para o avanço das práticas de saúde pública.

DESCRITORES: Cuidadores; Deficiência Intelectual; Enfermagem; População Rural; Apoio Social.

\section{CUIDADORES A DOMICILIO DE PERSONAS CON DISCAPACIDAD INTELECTUAL EN EL CONTEXTO RURAL Y SUS RETOS}

\section{RESUMEN:}

Objetivo: analizar los retos, características y sentimientos de los cuidadores domiciliarios de personas con discapacidad intelectual en el contexto rural. Material y método: se trata de un estudio cuanti-cualitativo, con 124 cuidadores de personas con discapacidad intelectual que viven en un contexto rural, en ocho municipios del sur de Brasil. Los datos se recopilaron de septiembre de 2018 a junio de 2019, mediante un cuestionario con variables sociales, demográficas y cotidianas del cuidado. El análisis fue de tipo analítico y descriptivo, a través de la comparación de frecuencias. Resultados: los cuidadores domiciliarios son mujeres, madres, hijas, con edad avanzada, poca escolaridad, pero que, debido a los desafíos como el acúmulo de funciones, la sobrecarga de trabajo, la baja escolaridad, el cansancio, el aislamiento social y profesional, desenvuelven sus atribuciones amorosas. Conclusión: el estudio contribuyó a identificar las características y los desafíos de los cuidadores de personas con discapacidad intelectual en las zonas rurales, revelando cuestiones para el avance de las prácticas de salud pública.

Palabras clave: Cuidadores; Discapacidad Intelectual; Enfermería; Población Rural; Apoyo Social.

Recebido em: 31/03/2020

Aprovado em: 18/05/2021

Editora associada: Susanne Elero Betiolli

Autor Correspondente:

Darielli Gindri Resta Fontana

Universidade Federal de Santa Maria - Palmeira das Missões, RS, Brasil

E-mail: darielliresta@gmail.com

Contribuição dos autores:

Contribuições substanciais para a concepção ou desenho do estudo; ou a aquisição, análise ou interpretação de dados do estudo - Silva TB de Q da; Elaboração e revisão crítica do conteúdo intelectual do estudo - Silva TB de Q da; Responsável por todos os aspectos do estudo, assegurando as questões de precisão ou integridade de qualquer parte do estudo - Fontana DGR, Jantsch LB, Colomé IC dos S, Costa MC da. Todos os autores aprovaram a versão final do texto.

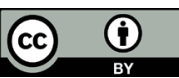

Copyright @ 2021 Este é um artigo em acesso aberto distribuído nos termos da Licença Creative Commons Atribuição, que permite o uso irrestrito, a distribuição e reprodução em qualquer meio desde que o artigo original seja devidamente citado. 